\title{
Widespread reduction in sleep spindle activity in socially anxious children and
} adolescents

Ines Wilhelm ${ }^{1,2,3}$, Sabine Groch ${ }^{1}$, Andrea Preiss ${ }^{4}$, Susanne Walitza ${ }^{4}$ and ${ }^{*}$ Reto Huber ${ }^{1,4}$

\author{
${ }^{1}$ University Children's Hospital Zürich, Switzerland \\ ${ }^{2}$ Department of Psychiatry, Psychotherapy and Psychosomatics, Psychiatric Hospital, \\ University of Zürich, Switzerland. \\ ${ }^{3}$ Department of Experimental Psychopathology and Psychotherapy, University of Zürich, \\ Switzerland. \\ ${ }^{4}$ Department of Child and Adolescent Psychiatry and Psychotherapy, Psychiatric Hospital, \\ University of Zürich, Switzerland
}

Address correspondence: Reto Huber, Steinwiessstrasse 75, 8032 Zürich, Switzerland;

Email: reto.huber@kispi.uzh.ch, Tel.: +41-(0)44-2663217, Fax: +41-(0)44-2667866 


\begin{abstract}
Social anxiety disorder (SAD) is one of the most prevalent psychiatric diseases typically emerging during childhood and adolescence. Biological vulnerabilities such as a protracted maturation of prefrontal cortex functioning together with heightened reactivity of the limbic system leading to increased emotional reactivity are discussed as factors contributing to the emergence and maintenance of SAD. Sleep slow wave activity (SWA, 0.75-4.5 Hz) and sleep spindle activity $(9-16 \mathrm{~Hz})$ reflect processes of brain maturation and emotion regulation. We used high-density electroencephalography to characterize sleep SWA and spindle activity and their relationship to emotional reactivity in children and adolescents suffering from SAD and healthy controls (HC). Subjectively rated arousal was assessed using an emotional pictureword association task. SWA did not differ between socially anxious and healthy participants. We found a widespread reduction in fast spindle activity $(13-16 \mathrm{~Hz})$ in SAD patients compared to HC. SAD patients rated negative stimuli to be more arousing and these arousal ratings were negatively correlated with fast spindle activity. These results suggest electrophysiological alterations that are evident at an early stage of psychopathology and that are closely linked to one core symptom of anxiety disorders such as increased emotional reactivity. The role of disturbed GABAergic neurotransmission is discussed as an underlying factor.
\end{abstract}




\section{Introduction}

Social anxiety disorder (SAD) is one of the most prevalent psychiatric diseases with a lifetime prevalence of up to 5-13\% of the general population (Essau et al. 1999; Furmark, 2002, Beesdo et al. 2007). On a clinical perspective, social anxiety is characterized by increased fear of negative evaluation in social situations and a tendency to avoid these kinds of situations. Besides psychological and environmental influences, biological vulnerabilities are discussed as critical factors for the development and maintenance of SAD (Clark and Wells, 2005; Rapee and Heimberg, 1997; Haller et al. 2014). A delayed maturation of prefrontal cortex functioning together with a heightened reactivity of limbic regions have been discussed as factors contributing to the increased emotional response to emotional stimuli in SAD patients compared to healthy individuals (Pfeifer and Blakemore, 2012; Haller et al. 2014). In line with that, subjectively rated arousal as well as arousal-induced amygdala activation in response to emotional stimuli was found to be increased in SAD patients as compared to healthy controls (Blair et al. 2011; Etkin and Wagner, 2007; Brühl et al. 2014; Wieser and Moscovitch, 2015). SAD typically emerges during the developmental phase of adolescence where essential cortical restructuring including the proliferation, migration, organization and myelination of neural circuits is taking place (Goldstein et al. 2009). Moreover, emotional reactivity is highest during adolescence (Merikangas et al. 2010; Blakemore and Choudhury, 2006; Paus et al. 2008; Haller et al. 2014). Thus, studying social anxiety during this developmental period can improve our understanding of the neurophysiological factors contributing to the emergence and maintenance of this mental disorder.

There is now accumulating evidence indicating that processes of brain maturation and emotion regulation are not only reflected in measures of neurophysiological activity during wakefulness but they are mirrored by oscillatory activity during sleep. Electrophysiological features such as sleep slow wave activity (SWA, EEG activity at a frequency of $0.75-4.5 \mathrm{~Hz}$ ) 
Wilhelm, I. et al.

and sleep spindle activity (EEG activity at a frequency of 9-16 Hz) that are characteristic of non-rapid eye movement sleep (NREM sleep) are of particular importance in this context. First evidence suggests a close relationship between SWA and processes of brain maturation. More specifically, SWA mirrors the extensive synaptic reorganization of cortical areas that take place from early childhood to late adolescence (Campbell and Feinberg, 2009; Kurth et al. 2010). SWA also reflects processes of use-dependent plasticity as SWA was found to be increased over those brain regions that had been extensively used during preceding wakefulness (Huber et al. 2004; Huber et al. 2006; Wilhelm et al. 2014). Sleep spindles, thalamo-cortically generated phasic oscillations, have been found to be related to brain plasticity in adults. For example, performing on a learning task resulted in an increase in spindle activity during the subsequent night and this increase was associated with the overnight improvement in task performance (Gais et al. 2002; Schabus et al. 2004). Spindle activity was also found to be positively associated with cognitive functioning such as intelligence or learning ability in adults (Schabus et al. 2006). First studies suggest developmental differences in the regulation and functioning of sleep spindles. For example, in a group of 8-11 years old children there was no increase in spindle activity after learning (Hödlmoser et al. 2014). Moreover, in contrast to adults, in children, the relationship between cognitive functioning and spindle activity showed conflicting results, i.e. positive and negative correlations (Chatburn et al. 2013; Geiger et al. 2011). Beside the role of sleep spindles in cognitive processes, spindles have been found to be related to emotion regulation abilities, stress response and coping strategies in children (Mikoteit et al. 2012; Mikoteit et al. 2013).

On the basis of these findings, it has been hypothesized that SWA as well as sleep spindle activity can reflect pathological processes of brain maturation and brain function that comes along with various psychiatric diseases (Tesler et al. 2013). First empirical evidence indeed supports this notion. Depressed adolescents compared to healthy controls showed 
Wilhelm, I. et al.

higher level of SWA over the prefrontal cortex and SWA over this cortical region was correlated with symptom severity in these patients (Tesler et al. 2015). The authors assumed that the increased prefrontal SWA is attributed either to accelerated processes of prefrontal maturation or to the increased rumination-induced use of the prefrontal cortex. ADHD patients exhibited increased SWA over central regions (Ringli et al. 2013) and this has been hypothesized to reflect the delayed maturation in these cortical regions. In schizophrenic patients and healthy controls with schizotypical personality traits, sleep spindle activity was found to be markedly reduced in a cluster of central electrodes (Ferarelli et al. 2007; Ferarelli et al. 2010; Lustenberger et al. 2015). This reduction of sleep spindles was assumed to reflect the loss of integrity of the thalamocortical system and inefficient information processing which is characteristic in these patients (Lustenberger et al. 2015). A reduction in sleep spindle activity was also found in a group of children and adolescents with early onset depression (Lopez et al. 2010). Moreover, remarkable deficits in sleep-dependent consolidation of neutral and emotional memories have been reported in ADHD, schizophrenia, social anxiety and depression with SWA and/or sleep spindles being associated with these deficits (Prehn-Kristensen et al. 2014; Göder et al. 2008; Göder et al. 2013; Dresler et al. 2010; Groch et al. 2016). Here, we aimed to investigate 1) SWA and spindle activity in children and adolescents suffering from social anxiety disorder and 2) the relationship between SWA/ spindle activity and measures of emotional memory processing (i.e. emotional memory consolidation and emotional reactivity such as subjective ratings of arousal) using high-density EEG. This method allows us to measure EEG activity at a high temporal and spatial resolution to uncover possible topographical differences.

\section{Materials and methods}

\section{Participants}


Wilhelm, I. et al.

Fourteen socially anxious participants (mean \pm SEM: $14.02 \pm 0.54$ yrs; 9 females, 5 males) and fourteen age-matched healthy controls (mean \pm SEM: $13.89 \pm 0.49$ yrs; 7 females, 7 males; age-range: 9-17 yrs) participated in this study. Healthy participants were recruited via advertisements in schools and local newspapers and socially anxious participants were recruited at the Department of Child and Adolescent Psychiatry and Psychotherapy, Psychiatric Hospital, University of Zürich. SAD patients met the criteria for a social anxiety disorder according to the criteria of the DSM-V (American Psychiatric Association, 2013) evaluated by an experienced Child and Adolescent Psychiatrist. To confirm this initial clinical diagnosis, a clinical interview was administered by a second experienced Child and Adolescent Psychiatrist using the Mini-International Neuropsychiatric Interview ((M.I.N.I.KID), Sheehan et al. 1998). The „Children's Depression Rating Scale Revised“ ((CDRS-R); Keller et al. 2011; see Table 1 for results) was used to exclude prevalent comorbidity of depression in these patients. The M.I.N.I-KID was also administered in the group of healthy participants to exclude any mental disorder in these participants. Symptoms of social anxiety were assessed in all participants using two questionnaires, i.e. the „Sozialphobie und Angstinventar für Kinder“"((SPAIK); Melfsen et al. 2001, see Table 1 for results) as well as the „Social Anxiety Scale for Children Revised“-Child and Parents version ((SASC-R-D), Melfsen and Florin, 1997, see Table 1 for results). Interviews with all participants and their parents as well as standardized questionnaires ensured that the participants had no cognitive impairments, neurological or sleep disorders. Participants did not take any medication at the time of the experiment except for one socially anxious participant who received selective serotonin reuptake inhibitors. The ingestion of caffeine or alcohol was not allowed on experiment days. The intelligence quotient (IQ) was further assessed ((WISC-IV); Petermann and Petermann, 2011) in each participant. Participants with an IQ below 85 and above 135 were excluded. Socially anxious patients did not differ from healthy controls with regard to IQ ( $\mathrm{p}>0.06$, see Table 1 for results). All participants were asked for individual sleep habits, 
Wilhelm, I. et al.

i.e., usual time to go to bed, time getting up, etc. in order to schedule the night in the sleep laboratory in accordance with their usual sleep habits.

The study was carried out in accordance with the latest version of the Declaration of Helsinki and approved by the local ethics committee. The parents of the participants gave written informed consent before their childrens' participation. Additionally, all children and adolescents provided verbal assent. All participants received a voucher for reimbursement of expenses (the value of the voucher was $100 \mathrm{CHF}$ for the initial screening, both experimental nights and one additional test session). Three participants (one SAD patient, two healthy controls) did agree to stay only for the first night in the sleep laboratory and thus, for these participants, no data on the picture-word association task was available. Accordingly, for the correlation analyses of memory performance/ arousal ratings and EEG activity only 13 SAD ( 8 females, 5 males) and 12 healthy controls ( 5 females, 7 males) could be included.

\section{Procedure}

Participants spent two nights in the sleep lab being separated by at least one night at home. In both sessions, participants came to the sleep lab either $1 \mathrm{hr}$ (first night) or $2.5 \mathrm{hrs}$ (second night) before their usual bed time. After their arrival, the electrode net was placed to record nocturnal sleep during the night. In the second night, participants came to the sleep lab around $2.5 \mathrm{hrs}$ before participants' habitual bedtime. After placing the electrode net, they performed the picture-word association task which took place between $7-11 \mathrm{PM}$. The next morning (Test Session 1), participants were awakened at their habitual wake time. Testing started $\sim 45$ minutes later (i.e. between 7-11AM) to avoid any modulating effect of sleep inertia on recall performance. Recall performance was again tested one week later (Test Session 2). In both test sessions, memory recall and subjective ratings of arousal of the stimuli was tested. Test Session 2 was performed via telephone. 
Wilhelm, I. et al.

\section{Picture-word association task.}

The picture-word association task used in our study is a modification of a task previously used in studies on mental imagery (Holmes et al. 2008; Pictet et al. 2011) and is described in detail elsewhere (Groch et al. 2015). In short, the task includes a set of 120 (for participants older than $12.4 \mathrm{yrs}$ ) or 102 (for participants younger than $12.4 \mathrm{yrs}$ ) ambiguous photographs of common everyday life objects and scenes. A different number of stimuli in younger and older participants was chosen to ensure comparable encoding level of $50-80 \%$ in all participants (mean \pm SEM, negative stimuli - HC: $75.77 \pm 2.52 \%$, SAD: $73.66 \pm 2.62 \%$; positive stimuli HC: $73.25 \pm 3.67 \%$, SAD: $73.08 \pm 2.81 \%$ ). This is considered to be relevant because the level of encoding is assumed to modulate long-term memory consolidation (Creery et al. 2015). Pictures were paired with words that had been chosen so that their combination with the picture (e.g. a picture showing a person acting in a play in front of an audience) either suggests a positive (half of the pictures; e.g. "applause") or a negative outcome of the scene shown on the picture (e.g. "jeer"). Participants had to generate mental imageries about this event by vividly imagining themselves in the situation. We chose here mental imagery instead of merely presenting the stimuli because this has been reported to elicit a much stronger emotional response due to the greater personal relevance (Holmes et al. 2008). Memory performance was tested for all picture-word associations in a cued recall procedure (i.e. the picture was presented and participants were asked to recall the associated word) immediately after learning (Learning Session), the next morning (Test Session 1) and one week later (Test Session 2). We calculated the relative difference between correctly recalled words in Test Session 1/ Test Session 2 and the Learning Session (with retention performance in the learning session set to $100 \%$ ) as a measure of long-term memory consolidation.

After testing memory performance in Test Session 1 and 2, all pictures were again presented and participants were instructed to rate spontaneously and quickly on a 9-point dimensional scale how emotional they perceive the picture on the dimensions of arousal (i.e. 
Wilhelm, I. et al.

excitement, $1=$ not arousing at all, and $9=$ very arousing) and pleasantness (this data is not reported).

\section{Sleep EEG recordings and EEG power analysis}

Sleep in both nights was recorded using high-density sleep EEG (Electrical Geodesics Sensor Net for long-term monitoring, 128 channels, referenced to a vertex electrode) including, electromyographic and electrooculographic recordings. Data were sampled at $500 \mathrm{~Hz}(0.01-$ $200 \mathrm{~Hz})$. Offline, the EEG was bandpass filtered $(0.5-50 \mathrm{~Hz})$ and downsampled to $128 \mathrm{~Hz}$. The EEG was visually scored for sleep stages Wake, N1, N2, N3 and REM at frontal, central and occipital electrodes (20 s epochs) based on American Academy of Sleep Medicine standard criteria (Iber et al. 2007). Spectral analysis of consecutive $20 \mathrm{~s}$ epochs (fast Fourier transform routine, Hanning window, averages of five $4 \mathrm{~s}$ epochs, frequency resolution of $0.25 \mathrm{~Hz})$ was performed for three frequency bands of interests, i.e. SWA $(0.75-4.5 \mathrm{~Hz})$, slow spindle activity $(9-12 \mathrm{~Hz})$ and fast spindle activity $(13-16 \mathrm{~Hz})$. Importantly, the terms "slow spindle activity" and "fast spindle activity" refer to EEG power in the slow and fast spindle frequency range and unique spindle events were not analyzed in this study.

Here, we present sleep EEG data from the first night in the sleep lab because 1) this makes our data comparable to previous studies in our lab on sleep EEG topography in children and adolescents with and without mental disorders (see for example Tesler et al. 2015; Kurth et al. 2010; Wilhelm et al. 2014) and 2) in the second night, auditory stimuli were presented during the night which might have interacted with the sleep EEG (this data is being published elsewhere).

\section{Statistical analysis}

Statistical analysis of memory retention and subjective ratings of arousal was based on $2 \times 2 \times$ 2 analyses of variance (ANOVA) including the repeated measures factors 'valence' (positive, 
Wilhelm, I. et al.

negative) and 'time' (Test Session 1, Test Session 2) and the between-subjects factor 'group' (socially anxious patients, healthy controls). In case of a significant interaction, further $2 \times 2$ ANOVAs and/or post-hoc t-tests were calculated in order to elucidate the source of the interaction. EEG oscillatory activity at all electrodes for the three frequency bands of interest were compared between socially anxious and healthy participants using student's t-test. Pearson's correlation coefficients were calculated for 1) EEG oscillatory activity and symptom severity of social anxiety and 2) EEG oscillatory activity and memory retention/arousal ratings. To control for multiple comparisons, statistical nonparametric mapping (SnPM) using a suprathreshold cluster analysis was applied (Nichols and Holmes, 2002) as done in a number of previous studies (e.g. Tesler et al. 2015 ; Lustenberger et al. 2015; Huber et al. 2004, Kurth et al. 2010; Ringli et al. 2013).

\section{Results}

Sleep EEG oscillatory activity in socially anxious adolescents

Both groups slept equally well, except for socially anxious participants who spent more time in Non REM sleep stage 1 than healthy controls (see Table 2 for descriptives). We compared EEG activity in socially anxious and healthy participants in the three frequency bands of interest such as slow wave $(0.75-4.5 \mathrm{~Hz})$, slow spindle $(9-12 \mathrm{~Hz})$ and fast spindle activity (13$16 \mathrm{~Hz}$ ) across the entire night. Note that calculating EEG activity in the first hour of NREM sleep does reveal the same pattern of differences between SAD and healthy controls. Data on EEG activity in the first hour was therefore not presented. For all three frequency ranges, we found the typical topographical distribution that has been reported in previous experiments in this age-group (Kurth et al. 2010; Tesler et al. 2015; Fig. 1A,B for SWA; Fig. 1D,F for slow spindle activity; Fig. 1G,H for fast spindle activity). SWA did not differ between SAD patients and healthy controls (Fig. 1C). Because previously reported SWA differences between healthy controls and depressed patients (Tesler et al. 2015) as well as ADHD patients 
(Ringli et al. 2013) were based on normalized values of SWA (i.e. EEG activity at a single electrode being divided by mean EEG activity over all electrodes) we also analyzed possible differences in normalized SWA between SAD and healthy controls. Both groups did also not differ with regard to normalized values of SWA (data not shown). There were significant differences in the slow and fast spindle range. Socially anxious subjects showed reduced slow spindle activity in a cluster of electrodes being mainly located over the occipital and temporal cortex (Fig. 1F, all electrodes $\mathrm{p}<0.05$ in a significant cluster are indicated as white dots). Fast spindle activity was markedly reduced in socially anxious participants as compared to healthy controls in nearly all electrodes (Fig. 1I, all electrodes $\mathrm{p}<0.05$ in a significant cluster are indicated as white dots). To further characterize the relationship between slow and fast spindle activity and social anxiety, we calculated correlations analyses between EEG activity in these two frequency bands and the results in two questionnaires of social anxiety (i.e. SPAIK and SASC-R) in socially anxious participants. Symptom severity of social anxiety as indicated by the SPAIK was neither correlated with slow nor fast spindle activity. The SASC$\mathrm{R}$ is a self and a parent report measure that differentiates between the two major features of social anxiety such as „fear of negative evaluation“ and „social avoidance and distress“. Both subscales of the self report version of the SASC-R showed a negative correlation with fast spindle activity in a cluster mainly located over the right fronto-temporal cortex ("social avoidance and distress" SAD: 24 electrodes; "fear of negative evaluation": 18 electrodes). Because significant electrodes of both correlation analysis strongly overlapped (i.e. 17 electrodes were significant in both analyses), a sum score was calculated. This sum score was negatively correlated with fast spindle activity (Fig. 2A) and a substantial amount of the significant electrodes in this analysis overlapped with those electrodes that showed significant differences between socially anxious and healthy controls (see Fig. 2B for an exemplary scatterplot indicating the relationship between sum score and fast spindles activity at one of these overlapping electrodes, i.e. electrode 122). Although the parent report measure of the 
Wilhelm, I. et al.

SASC-R also showed negative correlations with fast spindle activity in a number of electrodes, none of the clusters survived the cluster correction procedure. Slow spindle activity was neither correlated with the two subscales nor the sum scores of the parents' report or the self -report.

\section{Associations of fast spindle activity and subjective ratings of emotional arousal}

In line with previous findings (e.g. Wieser and Moscovitch, 2015), socially anxious participants did per se rate the learnt stimuli to be more arousing than healthy controls (main effect of 'group' in a 2 ('time') x 2 ('valence') x 2 ('group') ANOVA: $F(1,23)=7.29 ; \mathrm{p}=$ 0.013). Although being marginally stronger for negative stimuli, this effect was evident for both kind of stimuli (for negative stimuli: main effect of 'group': $F(1,23)=9.62 ; p=0.005$; for positive stimuli: $\mathrm{F}(1,23)=4.82 ; \mathrm{p}=0.038$ in a 2 × 2 ANOVA; interaction 'valence' and 'group' $\mathrm{F}(1,23)=4.01 ; \mathrm{p}=0.057$ in a $2 \times 2 \times 2$ ANOVA; Fig. 3A). In socially anxious patients, the subjectively rated arousal of negative stimuli in the morning after encoding was negatively correlated with fast spindle activity in clusters of electrodes mainly spanning the centroparietal cortex (Fig. 3B, see Fig. 3C for an exemplary scatterplot for one of the significant electrodes $111, \mathrm{R}=-0.67, \mathrm{p}=0.012$ ).

\section{Associations of fast spindle activity and long-term consolidation of emotional memories}

Both groups did not differ in the long-term consolidation of positive and negative memories in the picture-word association task (all main effects and interactions including the factor 'group' $\mathrm{p}>0.37$; see Table 3 for descriptive data of retention performance in Test Session 1 and Test Session 2). In healthy controls but not in socially anxious participants, retention performance of negative memories in the morning after encoding was positively correlated with fast spindle activity in a cluster of twenty-four electrodes over the frontal cortex 
Wilhelm, I. et al.

( $\mathrm{p}<0.05$, SnPM, suprathreshold cluster test controlling for multiple comparisons, Nichols and Holmes, 2002).

\section{Discussion}

In this study, we compared oscillatory brain activity at a high temporal and spatial resolution in socially anxious children and adolescents and age-matched healthy controls. Slow wave activity (SWA) did not differ between the two groups but there was a profound reduction in slow and fast sleep spindle activity in SAD patients. Importantly, fast spindle activity was not only correlated with symptom severity of social anxiety but also with a major characteristic of social anxiety which is the increased emotional response to emotional stimuli.

A protracted maturation of the prefrontal cortex is assumed to be a key factor in the development of social anxiety disorder during adolescence (Pfeifer and Blakemore, 2012; Haller et al. 2014). Because SWA is a marker of local brain maturation and of use-dependent plasticity one could have expected a reduction in prefrontal SWA in SAD patients. In fact, prefrontal SWA tended to be lower on a descriptive level but it did not reach statistical significance. During late childhood and early adolescence, the prefrontal cortex undergoes profound structural changes (Sowell et al. 2002; Casey et al. 2005) that are also reflected in a steep decline in SWA (Kurth et al. 2010; Campbell and Feinberg, 2009). The wide age range (i.e., 9-17 years) and the small size of our sample leading to a great variability within our two groups might have hindered the detection of statistically significant differences. In order to be able to clarify whether differences in SWA between adolescents suffering from social anxiety and healthy controls exists or not, future studies should include a higher number of subjects with a narrow age-range.

Fast spindle activity was significantly correlated with the long-term consolidation of negative memories in healthy participants. There are several lines of research indicating a tight link between processes of memory formation and sleep spindles. It has been reported 
Wilhelm, I. et al.

that sleep spindles are implicated in the long-term consolidation of memories (for review see Rasch and Born, 2013) possibly by inducing neocortical plasticity (Timofeev et al. 2002; Rosanova and Ulrich, 2005). More specifically, learning a memory task was found to induce an increase in spindle activity in the post-learning night and this increase was also related to the overnight improvement in performance (Gais et al. 2002; Schabus et al. 2004). Besides these state-like aspects of spindle activity, there is accumulating evidence for a trait-like stability of spindle activity within an individual across multiple nights (Finelli et al. 2001; deGennaro et al. 2005). Such trait-like measures of spindles have been found to be related to general cognitive functioning, e.g. intellectual ability and learning capacity (Lustenberger et al. 2015; Bodizs et al. 2005; Schabus et al. 2006). Here, we measured spindle activity and memory retention on different sessions which precludes any conclusion on state-related aspects of the link between spindles and memory consolidation. The positive correlation between both measures rather indicates that trait-aspects are more relevant for this correlation. The fact that this correlation was only significant in the group of healthy subjects but not in SAD patients suggests that other factors affecting spindle activity such as the above mentioned GABAergic disturbances might have modulated the relationship between spindles and memory consolidation in these patients.

Our findings of reduced fast spindle activity in SAD patients as compared to healthy controls together with negative correlations between fast spindle activity and measures of social anxiety as indicated by questionnaires in the group of SAD patients indicates a close link between fast spindle activity to social anxiety. Although this study cannot clarify the underlying molecular mechanism behind this relationship, it is likely that the reduced fast spindle activity in social anxiety reflects the globally disturbed GABA-A neurotransmission in these patients (for review see Nuss, 2015). Sleep spindles are generated by close interactions between thalamocortical neurons and GABAergic inhibitory neurons of the reticular nucleus of the thalamus. Recent findings demonstrating that a drop in activity of 
Wilhelm, I. et al.

these inhibitory neurons coincided with the termination of a sleep spindle indicates a role of GABA neurotransmission in the generation of sleep spindles (Bartho et al. 2014). Moreover, benzodiazepines which are known to reduce symptoms of anxiety possibly due to their modulating impact on the GABA-A system (Lydiard et al. 2003; Nuss, 2015) was found to increase spindle activity (Plante et al. 2015; Aeschbach et al. 1994; Borbely et al. 1985; Dijk et al. 1989; Feige et al. 1999; Trachsel et al. 1990). This effect has been suggested to result from the potentiation of the thalamic reticular neurons (Plante et al. 2015). Our findings of increased arousal in SAD patients together with the negative correlation between arousal ratings and fast spindle activity fully support this argumentation. Benzodiazepines also resulted in a reduction of arousal-induced amygdala response towards emotional stimuli (Paulus et al. 2005; Del-Ben et al. 2012) indicating that the disturbed GABA-A system is also related to increased emotional reactivity in patients suffering from anxiety disorders. In sum, reduced fast spindle activity might mirror the disturbed GABA-A system in social anxiety and is therefore not only related to symptom severity but also to the increased emotional reactivity which is a well-described phenomena in these patients (Blair et al. 2011; Etkin and Wagner, 2007; Brühl et al. 2014; Wieser and Moscovitch, 2015). To further explore this idea, future studies should use MR spectroscopy in order to measure thalamic neurotransmission.

Participating in an experiment that includes cognitive testing as well as an overnight stay in a sleep laboratory is a huge challenge for socially anxious children and adolescents. On the one hand, this can explain the rather low number of participants who were in the end willing to participate while a much larger amount of patients (> 90 patients) were informed about the experiment but did not agree to participate. On the other hand, this challenging situation might have resulted in a selection of socially anxious participants with rather low to moderate symptom severity. The fact that we found a correlation between fast spindle activity and the SASC-R-D but not with the SPAIK might be related to this issue since the SASC-R-D in contrast to the SPAIK is assumed to assess rather subclinical level of social anxiety (Büch 
et al. 2015). However, we do not believe that a possible selection of socially anxious patients with relatively low level of symptom severity does challenge the interpretation of our findings. To the contrary, the global reduction in fast spindle activity we found in such a group of SAD patients confirms the potential magnitude of the effect. In more severely affected SAD patients we may expect even more pronounced differences.

This data set is part of a larger study including two nights of sleep in a sleep laboratory. Here, we analyzed EEG data from the first night because auditory stimuli were presented during the second night and this critically affects EEG activity (Groch et al. submitted for publication). Accordingly, one might argue that a first night effect possibly being even more pronounced in the group of socially anxious participants would have confounded our results. Despite of strong evidence in adult samples (e.g. Agnew et al. 1966) we are not aware of any study providing evidence for a first night effect in children and/or adolescents (see for example Hödlmoser et al. 2014 who reported in children comparable sleep parameters in the first and second night in a sleep lab). Additionally, when comparing sleep parameters between the first and the second night in our sample, we were also not able to find any signs for a first night effect. More specifically, none of the patterns typically associated with a first night effect such as an increase of time spent in sleep stage N1, time spent awake and sleep latency in the first as compared to the second night was observable in any of the two groups of participants (all p > 0.12). Accordingly, we are convinced that analyzing EEG spindle activity from the second night would have provided the same results as from the first night. However, future studies should analyze EEG activity after an adaptation night to confirm this argumentation

To sum up, our results indicate that measuring oscillatory activity during sleep at a high temporal and spatial resolution is useful in order to further characterize pathological processes of brain activity in mental disorders. This method is easy to apply, less expensive than MRI and is less affected by the modulating impact of conscious thoughts and cognitive 
Wilhelm, I. et al.

processes that are hardly controllable when measuring brain activity during the wake state. Our findings indicate that fast spindle activity is a useful marker of anxiety as it very likely reflects the disturbed GABAergic neurotransmission which is characteristic for this mental disorder. In order to further understand the relationship between GABA, anxiety and sleep spindles, future studies are needed that test 1) the generalizability of this effect by studying fast spindle activity in different populations of patients with different anxiety disorders and 2) the sensitivity of sleep spindle activity towards therapy-induced changes in anxiety. 


\section{References}

Agnew, H.W., Webb, W.B., Williams, R.L., 1966. The first night effect: an EEG study of sleep. Psychophysiology 2(3), 263-6.

Aeschbach, D., Dijk, D.J.., Trachsel, L., Brunner, D.P., Borbély, A.A., 1994. Dynamics of slow-wave activity and spindle frequency activity in the human sleep EEG: effect of midazolam and zopiclone. Neuropsychopharmacology 11, 237-244.

American Psychiatric Association, 2013. Diagnostic and statistical manual of mental disorders (5th ed.). Washington, DC.

Bartho, P., Slezia, A., Matyas, F., Faradzs-Zade, L., Ulbert, I., Harris, K.D., Laszlo, A., 2014. Ongoing network state controls the length of sleep spindles via inhibitory activity. Neuron 82 , $1367-1379$.

Beesdo, K., Bittner, A., Pine, D.S., Stein, M.B., Höfler, M., Lieb, R., Wittchen, H.U., 2007. Incidence of social anxiety disorder and the consistent risk for secondary depression in the first three decades of life. Arch Gen Psychiatry 64(8), 903-12.

Blair, K.S., Geraci, M., Korelitz, K., Otero, M., Towbin, K., Ernst, M., Leibenluft, E., Blair, R.J., Pine, D.S., 2011. The pathology of social phobia is independent of developmental changes in face processing. Am J Psychiatry 168(11),1202-1209.

Blakemore, S.J., Choudhury, S. 2006. Development of the adolescent brain: implications for executive function and social cognition. J Child Psychol Psychiatry 47, 296-312.

Bódizs, R., Kis, T., Lázár, A.S., Havrán, L., Rigó, P., Clemens, Z., Halász, P. 2005.

Prediction of general mental ability based on neural oscillation measures of sleep. J Sleep

Res. 14(3), 285-92.

Borbély, A.A., Mattmann, P., Loepfe, M., Strauch, I., Lehmann, D., 1985. Effect of benzodiazepine hypnotics on all-night sleep EEG spectra. Hum Neurobiol 4, 189-194.

Brühl, A.B., Delsignore, A., Komossa, K., Weidt, S., 2014. Neuroimaging in social anxiety disorder-a meta-analytic review resulting in a new neurofunctional model. Neurosci Biobehav Rev 47, 260-280.

Büch, H., Döpfner, M., Petermann, U., 2015. Soziale Ängste und Leistungsängste. Göttingen: Hogrefe.

Campbell, I.G., Feinberg, I., 2009. Longitudinal trajectories of non-rapid eye movement delta and theta EEG as indicators of adolescent brain maturation. Proc Natl Acad Sci USA 106, $5177-5180$.

Casey, B.J., Tottenham, N., Liston, C., Durston, S., 2005. Imaging the developing brain: what have we learned about cognitive development? Trends Cogn Sci 9, 104-110.

Chatburn, A., Coussens, S., Lushington, K., Kennedy, D., Baumert, M., Kohler,, M. 2013. Sleep spindle activity and cognitive performance in healthy children. Sleep 36(2), 237-43. 
Clark, D.M., Wells, A., 1995. A cognitive model of social phobia. In Heimberg, R.G et al. (Eds.), Social phobia: diagnosis, assessment, and Treatment. pp. 69-93. New York: Guilford.

Creery, J.D., Oudiette, D., Antony, J.W., Paller, K.A., 2015. Targeted Memory Reactivation during Sleep Depends on Prior Learning. Sleep 38(5), 755-63.

De Gennaro, L., Ferrara, M., Vecchio, F., Curcio, G., Bertini, M., 2005. An electroencephalographic fingerprint of human sleep. Neuroimage 26, 114-22.

Del-Ben, C.M., Ferreira, C.A., Sanchez, T.A., Alves-Neto, W.C., Guapo, V.G., de Araujo, D.B., Graeff, F.G., 2012. Effects of diazepam on BOLD activation during the processing of aversive faces. J Psychopharmacol 26(4), 443-451.

Dijk, D.J., Beersma, D.G., Daan, S., van den Hoofdakker, R.H., 1989. Effects of seganserin, a 5-HT2 antagonist, and temazepam on human sleep stages and EEG power spectra. Eur J Pharmacol 171, 207-218.

Dresler, M., Kluge, M., Genzel, L., Schüssler, P., Steiger, A., 2010. Impaired off-line memory consolidation in depression. Europ Neuropsychopharmacol 20, 553-561.

Essau, C.A., Conradt, J., Petermann, F., 1999. Frequency and comorbidity of social phobia and social fears in adolescents. Behav Res Ther 37(9), 831-843.

Etkin, A., Wager, T.D., 2007. Functional neuroimaging of anxiety: a meta-analysis of emotional processing in PTSD, social anxiety disorder, and specific phobia. Am J Psychiatry 164(10), 1476-1488.

Feige, B., Voderholzer, U., Riemann, D., Hohagen, F., Berger, M., 1999. Independent sleep EEG slow-wave and spindle band dynamics associated with 4 weeks of continuous application of short-half-life hypnotics in healthy subjects. Clin Neurophysiol 110, 19651974.

Ferrarelli, F., Huber, R., Peterson, M.J., Massimini, M., Murphy, M., Riedner, B.A., Watson, A., Bria, P., Tononi, G., 2007. Reduced sleep spindle activity in schizophrenia patients. Am J Psychiatry. 164(3), 483-492.

Ferrarelli, F., Peterson, M.J., Sarasso, S., Riedner, B.A., Murphy, M.J., Benca, R.M., Bria, P., Kalin, N.H., Tononi, G., 2010. Thalamic dysfunction in schizophrenia suggested by wholenight deficits in slow and fast spindles. Am J Psychiatry 167(11), 1339-1348.

Finelli, L.A., Achermann, P., Borbély, A.A., 2001. Individual 'fingerprints' in human sleep EEG topography. Neuropsychopharmacology 25, 57-62.

Furmark, T., 2002. Social phobia: overview of community surveys. Acta Psychiatr Scand 105(2), 84-93.

Gais, S., Mölle, M., Helms, K., Born, J., 2002. Learning-dependent increases in sleep spindle density. J Neurosci 22, 6830-6834.

Geiger, A., Huber, R., Kurth, S., Ringli, M., Jenni, O.G., Achermann, P., 2012. The sleep EEG as a marker of intellectual ability in school age children. Sleep 23, 93-7. 
Göder, R., Fritzer, G., Gottwald, B., Lippmann, B., Seeck-Hirschner, M., Serafin, I., Aldenhoff, J., 2008. Effects of olanzapine on slow wave sleep, sleep spindles and sleep related memory consolidation in schizophrenia. Pharmacopsychiatry 41, 92-99.

Göder, R., Baier, P.C., Beith, B., Baecker, C., Seeck-Hirschner, M., Junghanns, K., Marshall, L., 2013. Effects of transcranial direct current stimulation during sleep on memory performance in patients with schizophrenia. Schizophr Res 144, 153-154.

Goldstein, G., Panchalingam, K., McClure, R.J., et al. 2009. Molecular neurodevelopment: An in vivo31P-1H MRSI study. J Int Neuropsychol Soc 15, 671-83.

Groch, S., McMakin, D., Guggenbühl, P., Rasch, B., Huber, R., Wilhelm, I., 2016. Memory cueing during sleep modifies the interpretation of ambiguous scenes in adolescents and adults. Dev Cogn Neurosci 17, 10-18.

Groch, S., Zinke, K., Wilhelm, I., Born, J., 2015. Dissociating the contributions of slow-wave sleep and rapid eye movement sleep to emotional item and source memory. Neurobiol Learn Mem 122, 122-130.

Haller, S.P.W., Kadosh, K.C., Lau, J.Y.F., 2014. A developmental angle to understanding the mechanisms of biased cognitions in social anxiety. Front Hum Neurosci 7, 846.

Hödlmoser, K., Heib, D.P.J., Roell, J., Peigneux, P., Sadeh, A., Gruber, G., Schabus, M., 2014. Slow sleep spindle activity, declarative memory, and general cognitive abilities in children. Sleep 37(9), 1501-1512.

Huber, R., Ghilardi, M.F., Massimini, M., Tononi, G., 2004. Local sleep and learning. Nature 430, 78-81.

Huber, R., Ghilardi, M.F., Massimini, M., Ferrarelli, F., Riedner, B.A., Peterson, M.J., Tononi, G., 2006. Arm immobilization causes cortical plastic changes and locally decreases sleep slow wave activity. Nat Neurosci 9(9), 1169-76.

Holmes, E.A., Mathews, A., Mackintosh, B., Dalgleish, T., 2008. The causal effect of mental imagery on emotion assessed using picture-word cues. Emotion 8(3), 395-409.

Iber, C., Ancoli-Israel, S., Chesson, A., Quan, S.F. for the American Academy of Sleep Medicine Medicine, 2007. The AASM manual for the scoring of sleep and associated events: Rules, terminology and technical specifications (1st ed.). Westchester, IL: American Academy of Sleep Medicine.

Keller, F., Grieb, J., Ernst, M., Sprober, N., Fegert, J.M., Kolch, M., 2011. Children's Depression Rating Scale-Revised (CDRS-R): development of a German version and psychometric properties in a clinical sample. Z Kinder Jugendpsychiatr Psychother 39(3), 179-185.

Kurth, S., Ringli, M., Geiger, A., LeBourgeois, M., Jenni, O.G., Huber, R., 2010. Mapping of cortical activity in the first two decades of life: a high-density sleep electroencephalogram study. J Neurosci 30, 13211-13219. 
Lancel, M., 1999. Role of GABAA receptors in the regulation of sleep: initial sleep responses to peripherally administered modulators and agonists. Sleep 22(1), 33-42.

Lopez, J., Hoffmann, R., Armitage, R., 2010. Reduced sleep spindle activity in early-onset and elevated risk for depression. J Am Acad Child Adolesc Psychiatry 49, 934-943.

Lustenberger, C., O'Gorman, R.L., Pugin, F., Tushaus, L., Wehrle, F., Achermann, P., Huber, R., 2015. Sleep spindles are related to schizotypal personality traits and thalamic glutamine/glutamate in healthy subjects. Schizophr Bull 41, 522-31.

Lydiard, R.B., 2003. The role of GABA in anxiety disorders. J Clin Psychiatry 64, 21-27.

Melfsen, S., Florin, I., 1997. Ein Fragebogen zur Erfassung sozialer Angst bei Kindern (SASC-R-D). Kindheit und Entwicklung. 6:5.

Melfsen, S., Florin, I., Warnke, A., 2001. SPAIK: Sozialphobie- und -angstinventar für Kinder. Göttingen: Hogrefe.

Merikangas, K.R., He, J.P., Burstein, M., Swanson, S.A., Avenevoli, S., Cui, L., Benjet, C., Georgiades, K., Swendsen, J., 2010. Lifetime prevalence of mental disorders in U.S. adolescents: results from the National Comorbidity Survey Replication--Adolescent Supplement (NCS-A). J Am Acad Child Adolesc Psychiatry 49(10), 980-9.

Mikoteit, T., Brand, S., Beck, J., Perren, S., von Wyl, A., von Klitzing, K., HolsboerTrachsler, E., Hatzinger, M., 2012. Visually detected NREM Stage 2 sleep spindles in kindergarten children are associated with stress challenge and coping strategies. World J Biol Psychiatry 13(4). 259-268.

Mikoteit, T., Brand, S., Beck, J., Perren, S., von Wyl, A., von Klitzing, K., HolsboerTrachsler, E., Hatzinger, M., 2013. Visually detected nrem stage 2 sleep spindles in kindergarten children are associated with current and future emotional and behavioural characteristics. J Sleep Res 22, 129-136.

Nichols, T.E., Holmes, A.P., 2002. Nonparametric permutation tests for functional neuroimaging: a primer with examples. Hum. Brain Mapp. 15, 1-25.

Nuss, P., 2015. Anxiety disorders and GABA neurotransmission: a disturbance of modulation. Neuropsychiatr Dis Treat, 165-175.

Paulus, M.P., Feinstein, J.S., Castillo, G., Simmons, A.N., Stein, M.B., 2005. Dose-dependent decrease of activation in bilateral amygdala and insula by lorazepam during emotion processing. Arch General Psychiatry 62(3), 282.

Petermann, F., Petermann, U., 2011. Wechsler Intelligence Scale for Children - (German Version, WISC-IV) (P. F. \& P. U. Eds. 4th ed.). Frankfurt am Main: Pearson Assessment.

Pictet, A., Coughtrey, A.E., Mathews, A., Holmes, E.A., 2011. Fishing for happiness: the effects of generating positive imagery on mood and behaviour. Behav Res Ther 49(12), 885891. 
Paus, T., Keshavan, M., Giedd, J.N. 2008. Why do many psychiatric disorders emerge during adolescence? Nat Rev Neurosci 9, 947-957.

Pfeifer, J.H., Blakemore, S.J. 2012. Adolescent social cognitive and affective neuroscience: past, present, and future. Soc Cogn Affect Neurosci 7, 1-10.

Plante, D.T., Goldstein, M.R., Cook, J.D., Smith, R., Riedner, B.A., Rumble, M.E., Jelenchick, L., Tononi, G., Benca, R.M., Peterson, M.J., 2015. Effects of oral temazepam on spectral activity and sleep spindles during non-rapid eye movement sleep: A high-density EEG investigation. Eur Neuropsychopharmacol 25(10), 1600-1610.

Prehn-Kristensen, A., Munz, M.T., Göder, R., Wilhelm, I., Korr, K., Vahl, W., Wiesner, C.D., Baving, L., 2014. Transcranial Oscillatory Direct Current Stimulation During Sleep Improves Declarative Memory Consolidation in Children With Attention-deficit/hyperactivity Disorder to a Level Comparable to Healthy Controls. Brain Stimulation 7(6), 793-799.

Rapee, R.M., Heimberg, R.G., 1997. A cognitive-behavioral model of anxiety in social phobia. Behav Res Ther 35(8), 741-756.

Rasch, B., Born, J., 2013. About sleep's role in memory. Physiol Rev 93(2), 681-766.

Ringli, M., Souissi, S., Kurth, S., Brandeis, D., Jenni, O.G., Huber, R., 2013. Topography of sleep slow wave activity in children with attention-deficit/hyperactivity disorder. Cortex 49, 340-7.

Rosanova, M., Ulrich, D., 2005. Pattern-specific associative long-term potentiation induced by a sleep spindle-related spike train. J Neurosci. 25(41), 9398-405.

Schabus, M., Gruber, G., Parapatics, S., Sauter, C., Klösch, G., Anderer, P., Klimesch, W., Saletu, B., Zeitlhofer, J., 2004. Sleep spindles and their significance for declarative memory consolidation. Sleep 27(8), 1479-85.

Schabus, M., Hödlmoser, K., Gruber, G., Sauter, C., Anderer, P., Klösch, G., Parapatics, S., Saletu, B., Klimesch, W., Zeitlhofer, J., 2006. Sleep spindle-related activity in the human EEG and its relation to general cognitive and learning abilities. Eur J Neurosci. 23(7), 173846.

Sheehan, D.V., Lecrubier, Y., Sheehan, K.H., Amorim, P., Janavs, J., Weiller, E., Herqueta, T., Baker, R., Dunbar, G.C., 1998. The Mini-International Neuropsychiatric Interview (M.I.N.I.): the development and validation of a structured diagnostic psychiatric interview for DSM-IV and ICD-10. J Clin Psychiatry 59, 22-33.

Sowell, E.R., Trauner, D.A., Gamst, A., Jernigan, T.L., 2002. Development of cortical and subcortical brain structures in childhood and adolescence: a structural MRI study. Dev Med Child Neurol 44, 4-16.

Tesler, N., Gerstenberg, M., Huber, R., 2013. Developmental changes in sleep and their relationships to psychiatric illnesses. Curr Opin Psychiatry 26, 572-9. 
Tesler, N., Gerstenberg, M., Franscini, M., Jenni, O.G., Walitza, S., Huber, R., 2015. Reduced sleep spindle density in early onset schizophrenia: a preliminary finding. Schizophr Res 166, $355-7$.

Tesler, N., Gerstenberg, M., Franscini, M., Jenni, O.G., Walitza, S., Huber, R., 2015. Increased frontal sleep slow wave activity in adolescents with major depression. Neuroimage Clin 10, 250-6.

Timofeev, I., Grenier, F., Bazhenov, M., Houweling, A.R., Sejnowski, T.J.,, Steriade, M., 2002. Short- and medium-term plasticity associated with augmenting responses in cortical slabs and spindles in intact cortex of cats in vivo. J Physiol. 542, 583-98.

Trachsel, L., Dijk, D.J., Brunner, D.P., Klene, C., Borbély, A.A., 1990. Effect of zopiclone and midazolam on sleep and EEG spectra in a phase-advanced sleep schedule. Neuropsychopharmacol 3, 11-18.

Wieser, M.J., Moscovitch, D.A., 2015. The Effect of Affective Context on Visuocortical Processing of Neutral Faces in Social Anxiety. Front Psychol, 6:1824.

Wilhelm, I., Kurth, S., Ringli, M., Mouthon, A.L., Buchmann, A., Geiger, A., Jenni, O.G., Huber, R., 2014. Sleep slow-wave activity reveals developmental changes in experiencedependent plasticity. J Neurosci 34, 12568-12575. 
Wilhelm, I. et al.

\section{Figure legends}

Fig. 1 EEG power in socially anxious patients and healthy controls. Topographical distribution of EEG power during nocturnal sleep in socially anxious participants (SAD; A,D, G) and healthy controls (HC; B,E,H) in three frequency bands of interest such as SWA (0.54.5Hz; A,B), slow spindle activity $(9-12 \mathrm{~Hz} ; \mathrm{D}, \mathrm{E})$ and fast spindle activity $(13-16 \mathrm{~Hz} ; \mathrm{G}, \mathrm{H})$. Topographical distribution of the difference between SAD and HC for SWA (C), slow spindle activity (F) and fast spindle activity (I). Colour-coded T-values of unpaired t-tests for each electrode are indicated. Electrodes that show significant differences $(p<0.05$, SnPM, suprathreshold cluster test controlling for multiple comparisons, Nichols and Holmes, 2002) are indicated as white dots. Slow and fast spindle activity was significantly reduced in SAD compared to $\mathrm{HC}$ over multiple cortical regions.

Fig. 2 Correlation between symptom severity and fast spindle activity in SAD patients. Topographical distribution of the correlation between fast spindle activity and symptoms of social anxiety in SAD patients as indicated by the self-report version (A) of the SCRS-D. The colour coded correlation coefficients for each electrode are indicated. Significant electrodes ( $p<0.05$, SnPM, suprathreshold cluster test controlling for multiple comparisons, Nichols and Holmes, 2002) are indicated as white dots. In multiple electrodes, fast spindle activity was negatively correlated with symptom severity. Scatterplots visualizes the negative correlation between fast spindle activity and symptom severity (B) at an exemplary electrode (electrode 122 which is indicated by red circles in A).

Fig. 3 Arousal ratings and fast spindle activity. Arousal ratings of positive and negative pictures in Test Session 1 and Test Session 2 are higher in socially anxious (white bars) as 
Wilhelm, I. et al.

compared to healthy controls (black bars). The main effect in a 2 (time) x 2 (valence) x 2 (group) is indicated. Topographical distribution of the correlation between fast spindle activity and arousal ratings for negative stimuli at Test Session 1 (B). The colour coded correlation coefficients for each electrode is indicated. Significant electrodes $(p<0.05$, SnPM, suprathreshold cluster test controlling for multiple comparisons, Nichols and Holmes, 2002) are indicated as white dots. Scatterplots indicate the negative correlation between fast spindle activity and subjective ratings of arousal for an exemplary electrode $(\mathrm{C}$, electrode 111 which is indicated by a red circle in B). 
Table 1 Participants' data

\begin{tabular}{lcc}
\hline & $\begin{array}{c}\text { Healthy Controls } \\
\text { mean } \pm \text { SEM }\end{array}$ & SAD \\
& $13.89 \pm 0.49$ & $14.02 \pm 0.54$ \\
\hline Age & $107.43 \pm 3.28$ & $116.37 \pm 3.22$ \\
Intelligence Quotient & & \\
Questionnaires & $15.57 \pm 0.97$ & $25.93 \pm 2.53^{* *}$ \\
SASC-R-D FNE & $17.64 \pm 1.05$ & $32.79 \pm 2.09^{* * *}$ \\
SASC-R-D SAD & $13.14 \pm 0.86$ & $28.69 \pm 2.26^{* * *}$ \\
SASC-R-D FNE/ Parents & $15.86 \pm 1.18$ & $31.46 \pm 2.35^{* * *}$ \\
SASC-R-D SAD/ Parents & $7.26 \pm 1.00$ & $27.43 \pm 3.56^{* * *}$ \\
SPAIK & & $27.00 \pm 3.14$ \\
CDRS-R & &
\end{tabular}

All parameters are given in mean $( \pm$ SEM) and statistical differences between groups are indicated $(* * \mathrm{p}<0.01 ; * * * \mathrm{p}<0.001)$. 
Table 2 Memory retention

$$
\begin{array}{cc}
\text { Healthy Controls } & \text { SAD } \\
\text { mean } \pm \text { SEM } & \text { mean } \pm \text { SEM }
\end{array}
$$

Test session 1

$\begin{array}{lll}\text { Positive } & 102.90 \pm 1.66 & 101.37 \pm 1.52 \\ \text { Negative } & 100.36 \pm 1.14 & 100.87 \pm 1.72\end{array}$

Test session 2

$\begin{array}{lll}\text { Positive } & 88.58 \pm 2.94 & 86.39 \pm 2.15 \\ \text { Negative } & 87.67 \pm 3.80 & 83.38 \pm 2.88\end{array}$

Memory retention is given in percent relative to baseline learning. All parameters are given in mean ( \pm SEM) 
Table 3 Sleep parameters

\begin{tabular}{lcccc}
\hline & \multicolumn{2}{c}{ Absolute time (in min) } & \multicolumn{2}{c}{ Percentage of TST (in \%) } \\
\cline { 2 - 5 } & Healthy Controls & SAD & Healthy Controls & SAD \\
& mean \pm SEM & mean \pm SEM & mean \pm SEM & mean \pm SEM \\
\hline Sleep latency & $22.07 \pm 6.56$ & $21.62 \pm 3.84$ & & \\
REMS latency & $180.12 \pm 12.15$ & $184.71 \pm 19.34$ & & \\
Wake Time & $46.10 \pm 9.21$ & $62.57 \pm 13.23$ & & \\
Waso & $25.21 \pm 3.90$ & $44.17 \pm 11.45$ & & $5.98 \pm 0.94 * *$ \\
Non REMS 1 & $11.62 \pm 1.99$ & $31.33 \pm 4.91 * *$ & $2.49 \pm 0.46$ & $42.86 \pm 2.50$ \\
Non REMS 2 & $204.79 \pm 14.89$ & $226.29 \pm 15.86$ & $43.29 \pm 2.89$ & $32.04 \pm 2.61$ \\
Non REMS 3 & $176.12 \pm 12.79$ & $166.95 \pm 13.52$ & $36.90 \pm 2.29$ & $19.12 \pm 1.22$ \\
REMS & $84.19 \pm 10.17$ & $100.79 \pm 7.85$ & $17.31 \pm 1.67$ & \\
TST & $476.71 \pm 19.72$ & $525.36 \pm 17.07 \#$ & & \\
Awakenings & $29.50 \pm 3.76$ & $29.64 \pm 3.46$ & & \\
Stage Shifts & $165.07 \pm 11.96$ & $173.57 \pm 11.35$ & & \\
\hline Step pan & & & & \\
\hline
\end{tabular}

Sleep parameters for healthy and socially anxious (SAD) participants are given in mean \pm SEM of absolute time in minutes and percentage of total sleep time; REMS = rapid eye movement sleep, TST $=$ total sleep time. Statistical differences between age-groups are indicated $(\# p<0.10$; ** $p \leq 0.01)$. 
SAD

EEG power $\left(\mu \mathrm{V}^{2}\right)$

A

$0.75-4.5 \mathrm{~Hz}$

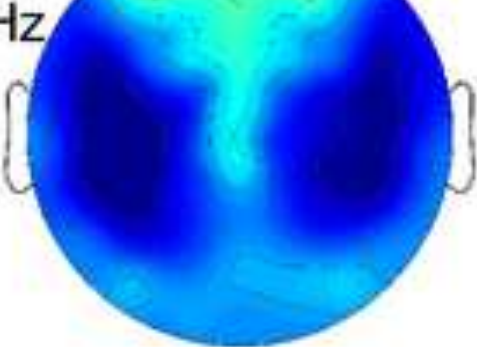

D

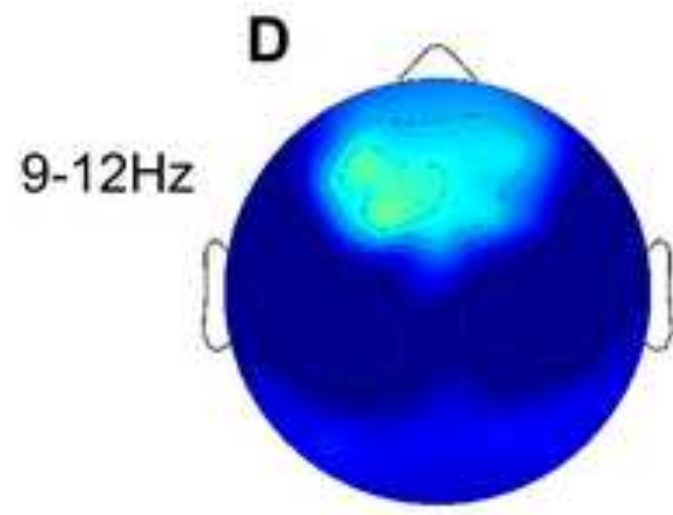

G

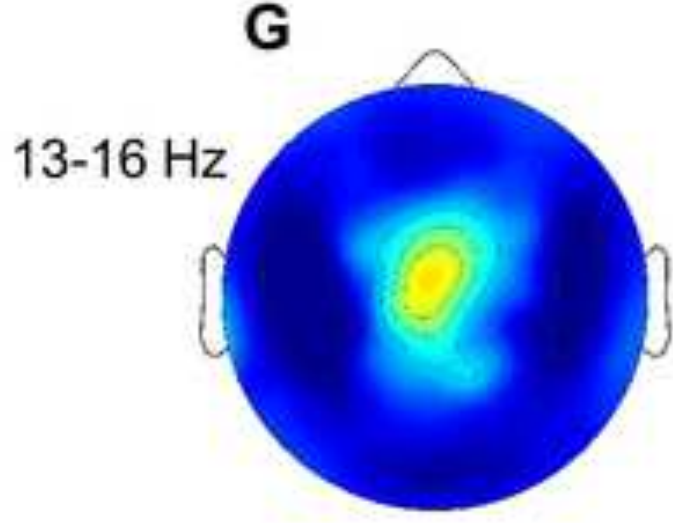

HC

EEG power $\left(\mu \mathrm{V}^{2}\right)$

B

350

300

250

200

150

100
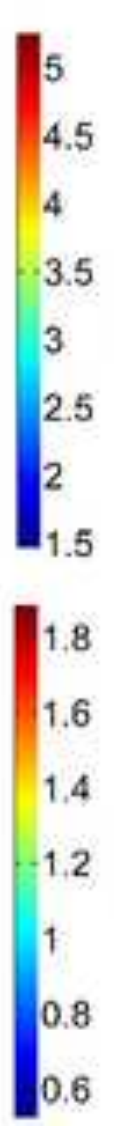

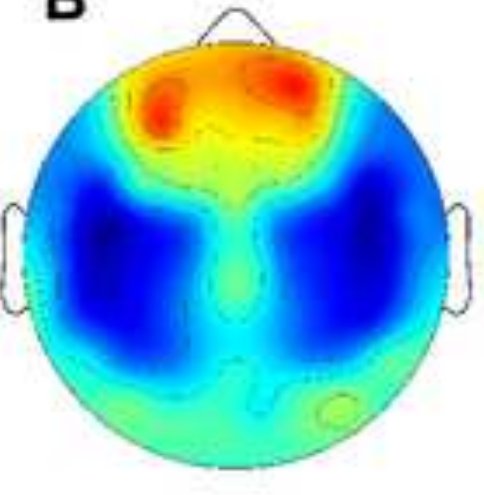

E

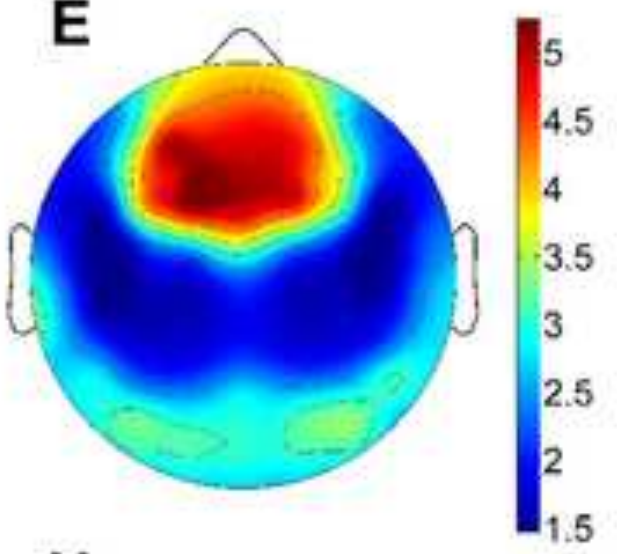

H

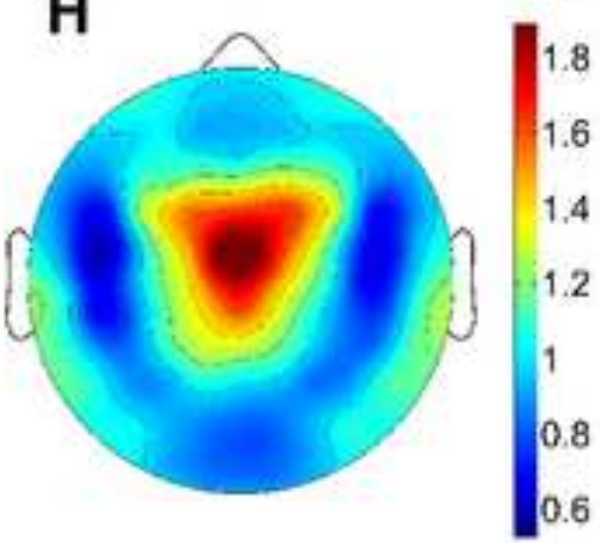

SAD/HC

T-values
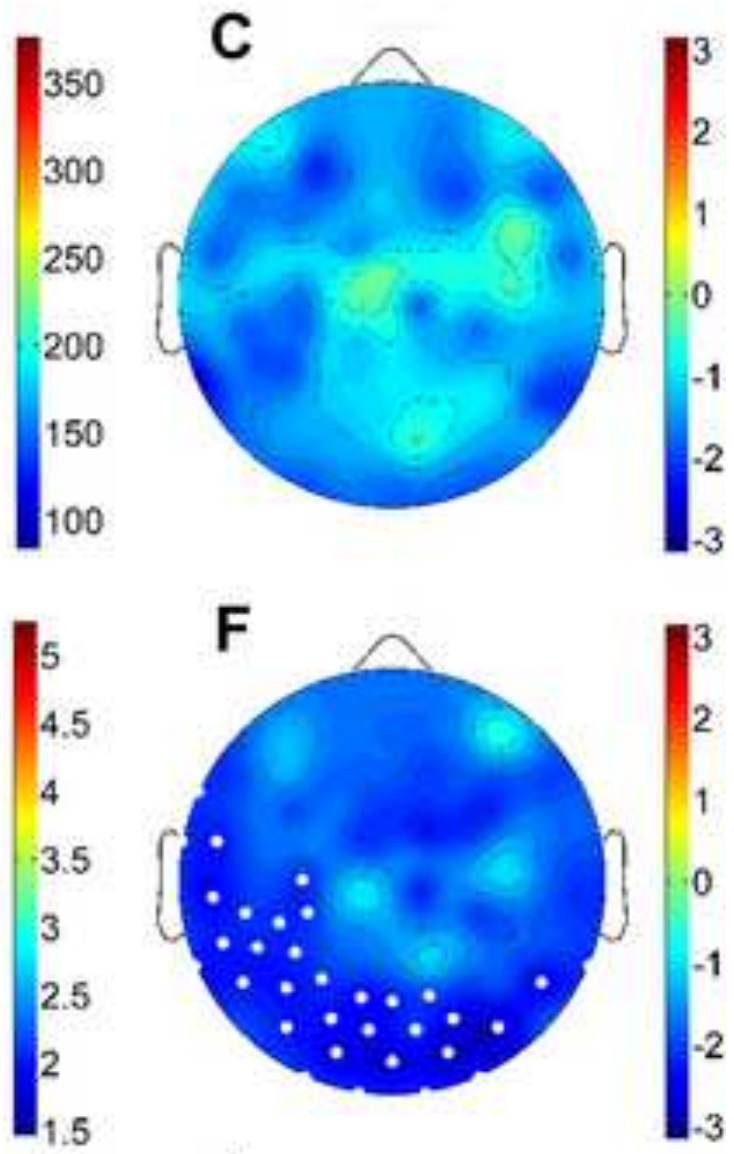

I

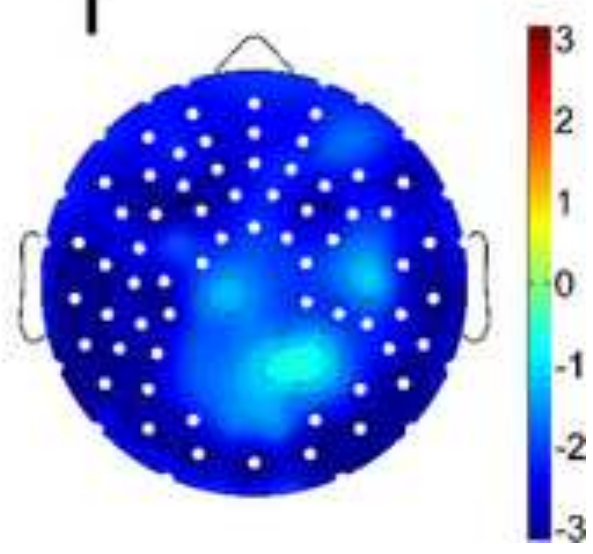


A

SCRS-K

B

\section{R-values}
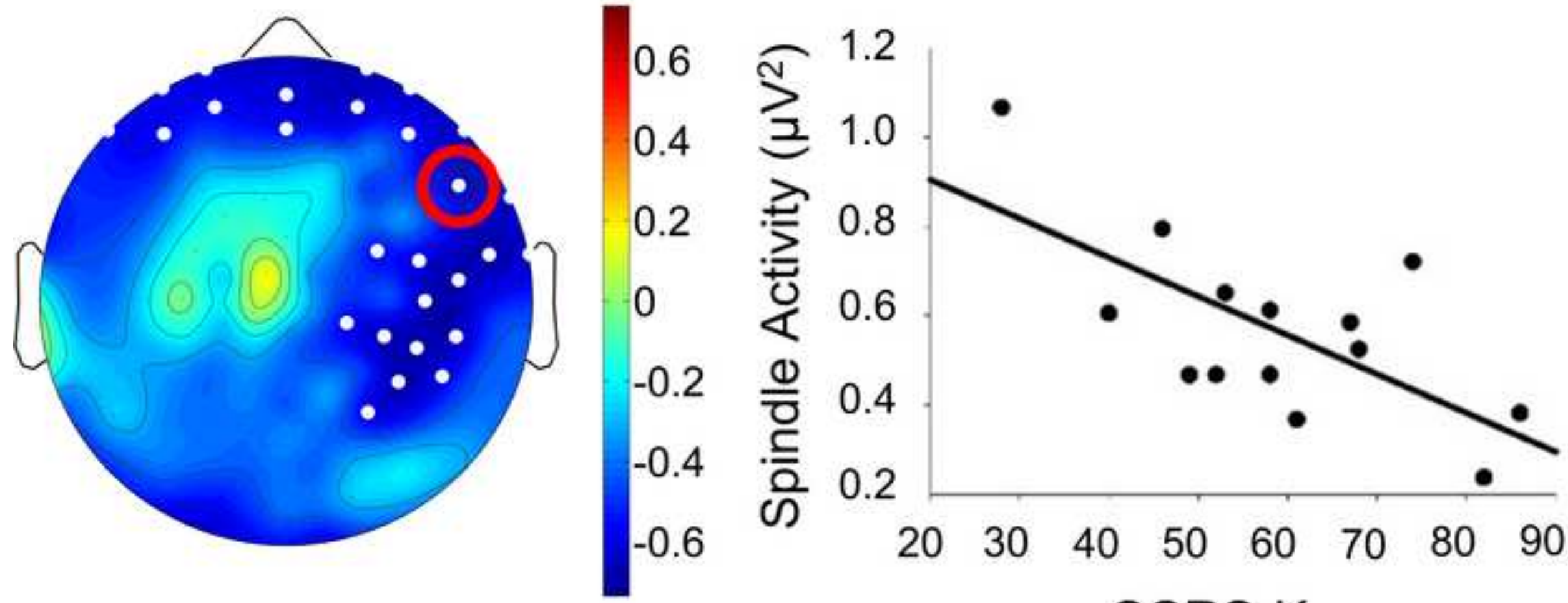

SCRS-K 
A

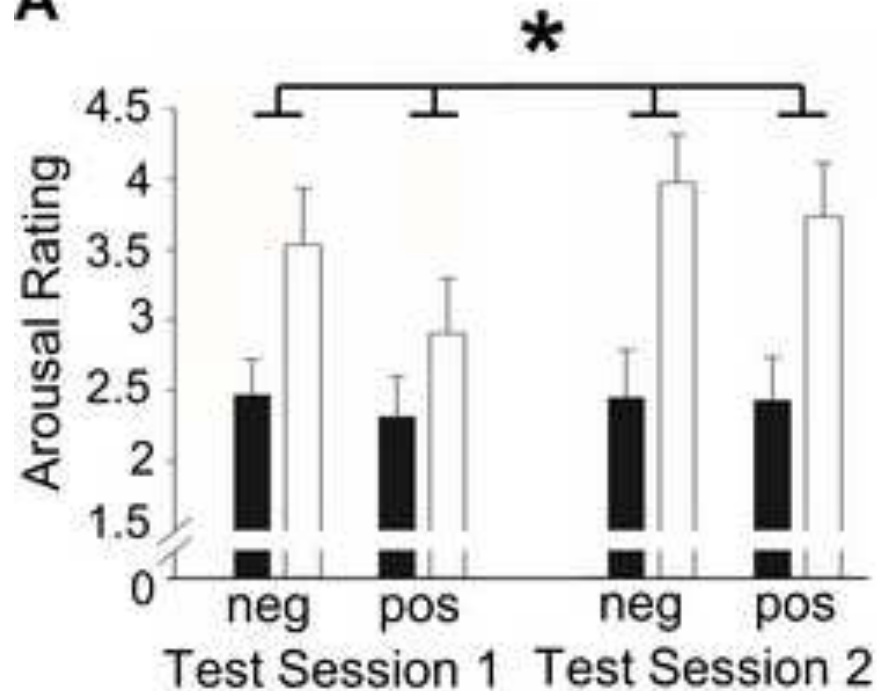

Test Session 1 Test Session 2

Healthy Controls
B

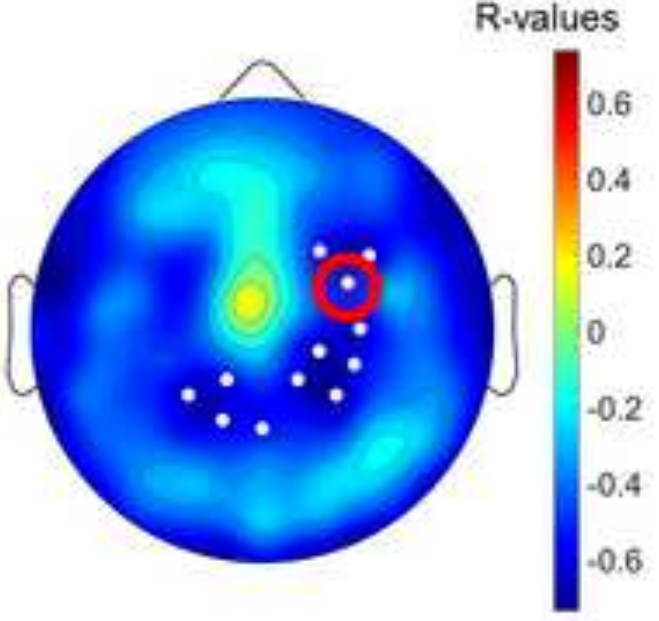

SAD
C

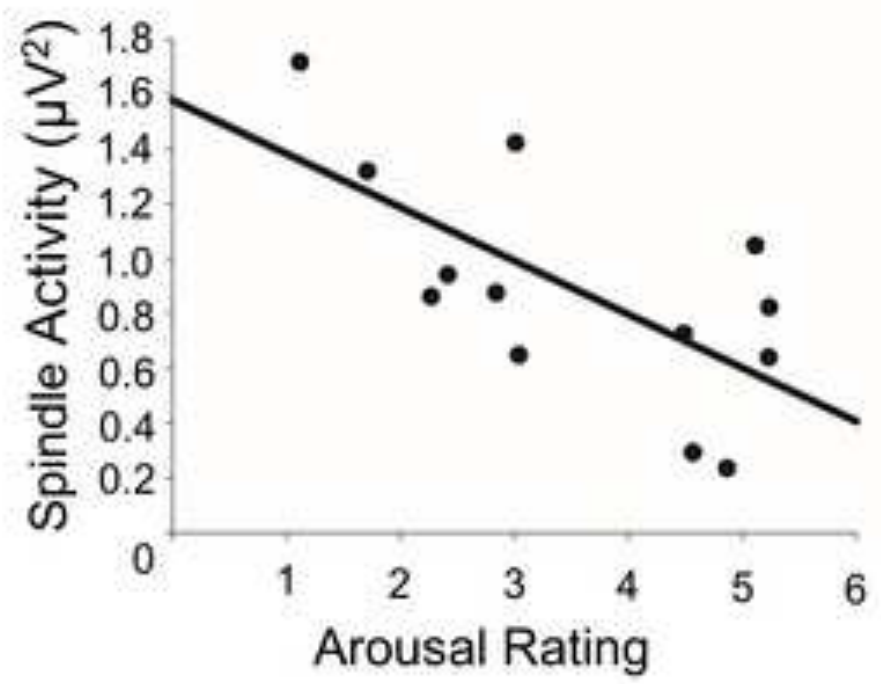




\section{Role of the funding sources}

All funding sources were not involved in the study design, collection, analysis and interpretation of the data, in writing the manuscript and in the decision to submit the article for publication. 


\section{Contributors}

All authors planned the experiment, IW, SG and AP conducted the experiment, IW and SG analysed the data, IW, SG and RH wrote the manuscript. 


\section{Acknowledgments}

The authors are highly grateful to Aylin Yantaz and Sina Unseld for their help with data collection. This work was supported by the Swiss National Science Foundation (Nr. 320030_153387), the Jacobs Foundation, the Child Research Centre of the University Children's Hospital, Zürich, the University of Zürich (Forschungskredit) and the CRPP Sleep and Health. 